\title{
Optical coherent control in semiconductors: Fringe contrast and inhomogeneous broadening
}

Østergaard, John Erland; Vadim, Lyssenko; Hvam, Jørn Märcher

Published in:

Physical Review B Condensed Matter

Link to article, DOI:

10.1103/PhysRevB.63.155317

Publication date:

2001

Document Version

Publisher's PDF, also known as Version of record

Link back to DTU Orbit

Citation (APA):

Østergaard, J. E., Vadim, L., \& Hvam, J. M. (2001). Optical coherent control in semiconductors: Fringe contrast and inhomogeneous broadening. Physical Review B Condensed Matter, 63(15), 155317.

https://doi.org/10.1103/PhysRevB.63.155317

\section{General rights}

Copyright and moral rights for the publications made accessible in the public portal are retained by the authors and/or other copyright owners and it is a condition of accessing publications that users recognise and abide by the legal requirements associated with these rights.

- Users may download and print one copy of any publication from the public portal for the purpose of private study or research.

- You may not further distribute the material or use it for any profit-making activity or commercial gain

- You may freely distribute the URL identifying the publication in the public portal 


\title{
Optical coherent control in semiconductors: Fringe contrast and inhomogeneous broadening
}

\author{
J. Erland, V. G. Lyssenko, and J. M. Hvam \\ Research Center COM, Technical University of Denmark, Oersteds Plads 349, DK-2800 Kgs. Lyngby, Denmark
}

(Received 14 August 2000; revised manuscript received 9 November 2000; published 30 March 2001)

\begin{abstract}
Optical coherent control experiments in semiconductors reveal how inhomogeneous broadening must be taken into account in contrast to previous coherent control experiments in atomic and molecular systems. With spectral resolution of the coherent control signal, the optical phases involved in the interplay between the homogeneous and inhomogeneous broadenings are measured. Based on these experiments, a coherent control model describing the optical fringe contrast using different detection schemes, such as photoluminescence or four-wave mixing, is established. Significant spectral modulation is observed in the four-wave mixing spectra as a function of phase-delay representing coherent control in the spectral domain. The spectral phase change of this modulation provides a spectroscopic tool to analyze contributions of inhomogeneous broadening to electronic resonances in semiconductor structures.
\end{abstract}

DOI: 10.1103/PhysRevB.63.155317

PACS number(s): 78.47.+p, 71.35.Cc, 42.25.Kb

\section{INTRODUCTION}

The developments with coherent control techniques in optical spectroscopy have recently demonstrated population control and coherence manipulations when the induced optical phase is explored with phase-locked laser pulses. Fascinating demonstrations were obtained with atoms and molecules such as localization of electronic wave packets in atomic sodium ${ }^{1}$ and control of molecular chemical reactions. ${ }^{2,3}$ More recently, the full characterization of the amplitude and phase of an electronic Rydberg wave packet in Cs (Ref. 4) was obtained using quantum state holography with tailored laser pulses. ${ }^{5}$ These developments have been guiding the new research field of quantum control and also the recent applications of coherent control techniques to semiconductors and nanostructures.

Population and orientation control of excitons in GaAs quantum wells and quantum dots, as well as control of electron-phonon scattering is GaAs, have provided advancements in ultrafast spectroscopy of semiconductors. ${ }^{6-9}$ In experiments recording the secondary emission, after resonant excitation with phase-locked pulses, with a spectral filtering technique ${ }^{10}$ or time-resolved ${ }^{7,11}$ using up-conversion, the coherent emission due to resonant Rayleigh scattering with optical fringes could be distinguished from incoherent photoluminescence without fringes. In the interpretation of these experiments in Gurioli et al. ${ }^{10}$ and Marie et al. ${ }^{7}$ and other experiments based on differential reflection measurements by Heberle et al. ${ }^{6}$ the exponential decay of the fringe contrast has been assigned to the homogeneous polarization decay with decay time $T_{2}$ in an analogy with the concepts developed in atomic and molecular spectroscopy. This, however, neglects the inhomogeneous broadening in semiconductor samples due to stochastic alloy and size fluctuations. On the other hand, Woerner and Shah ${ }^{11}$ and Garro et al. ${ }^{12}$ state that, in general, it is not possible to interpret the decay of the coherent control in terms of $T_{2}$. Woerner and Shah have experimentally observed an almost exponential decay of the fringe contrast versus delay between two pulses and ascribed this to the Fourier transform of the "real" exciton absorption line. In virtue of this, they come to the conclu- sion, that the measured dependence does not contain information about whether the investigated transition is homogeneously or inhomogeneously broadened. Moreover, in a number of recent papers by Garro et al. ${ }^{12}$ the experimentally measured coherent control (CC) signals have been compared with three-level optical Bloch calculations, taking into consideration the inhomogeneous broadening of excited levels while no dephasing was included. On the basis of such a comparison, these authors come to the conclusion that the $\mathrm{CC}$ decay is inversely proportional to the inhomogeneous broadening. These obvious contradictions in the interpretation of similar $\mathrm{CC}$ results can be resolved by detecting phase-dependent spectrally resolved four-wave mixing (FWM) that demonstrates how inhomogeneous broadening contributes to the phase dynamics.

In this paper, we have developed an improved basis for optical coherent control in semiconductors, taking both homogeneous and inhomogeneous broadening into account. We demonstrate the important influence from even small inhomogeneous broadenings, comparable to the homogeneous broadening, in spectrally resolved or spectrally integrated recordings of the coherent emission in coherent control experiments. Our model for the optical fringe contrast, developed on the basis of these experiments, succesfully describes, in a simple way, the spectrally resolved detection of the coherent emission, as well as the detection of time-integrated photoluminescence used in most experiments. We make appropriate calculations to deduce information about the dependence of spectral FWM oscillations on the amplitude ratio of the two phase-locked pulses, including homogeneous and inhomogeneous broadening of the excited states. We show that the significant interference effects in the FWM spectra have a simple dependence on the homogeneous broadening, as well as on the inhomogeneous broadening. Experimental examples from a narrow GaAs quantum well sample is given, demonstrating how the extracted phase information relates to the nature of the electronic resonances. This spectroscopy technique in semiconductor optics, using the phase information in coherently excited ensembles, is very important for extracting information on electronic dynamics in nanostructures and for coherent manipulations of populations, such as 


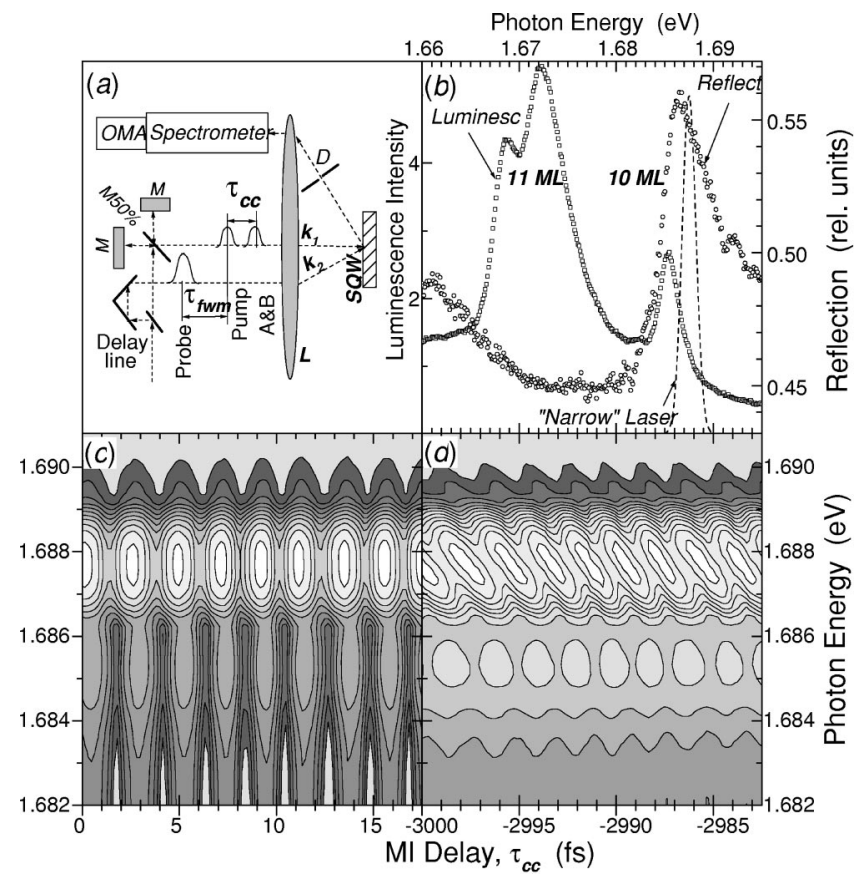

FIG. 1. (a) Experimental setup with Michelson interferometer for phase-locked pulse pair with time-delay $\tau_{c c}$. The four-wave mixing, generated by a time-delayed $\left(\tau_{f w m}\right)$ third pulse, is spectrally resolved and detected with an optical multichannel analyzer (OMA). (b) Luminescence (squares) and reflection (circles) spectra of the GaAs SQW revealing two regions with a thickness of 11 and 10 monolayers. The pump laser spectrum is shown dashed. (c,d) FWM spectra at $12 \mathrm{~K}$ with photoluminescence $(\hbar \omega<1.686 \mathrm{eV})$ for different phase delay round $\tau_{c c}=0 \mathrm{ps}$ and $\tau_{c c}=-3$ ps with $\tau_{f w m}$ $=0.5 \mathrm{ps}$.

those demonstrated previously in single quantum dot spectroscopy. ${ }^{8}$

\section{EXPERIMENTAL TECHNIQUE}

Our coherent control (CC) experiments are performed in a configuration where the photoluminescence (PL) and the coherent emission in four-wave mixing (FWM) are recorded after resonant excitation with phase-locked laser pulses with wave vector $k_{1}$. For the latter, a second beam with wave vector $k_{2}$ is incident on the sample resulting in the coherent scattering of the emission in the direction $2 k_{2}-k_{1}$. The experimental setup is illustrated in Fig. 1(a). Pulses from a 150 fs $80 \mathrm{MHz}$ Ti:Sapphire laser are split by a 30\% mirror into the "pump" and "probe" beams, the latter traveling through a delay line acquiring a time delay $\tau_{f w m}$ in the FWM, and focused on the sample surface by a lens $L$. The pump and probe beams have comparable intensities in the range of $0.1-1 \mathrm{~mW}$ with a spot diameter $\sim 100 \mu \mathrm{m}$ giving exciton densities of $\sim 10^{9} \mathrm{~cm}^{-2}$ for each pulse. All measurements were performed at two temperatures of 12 and $120 \mathrm{~K}$. The collinearly polarized phase-locked laser pump pulses, with an intensity ratio $a^{2}$, are generated in a Michelson interferometer (MI) with time delay $\tau_{c c}$. In contrast to most other CC experiments in semiconductors, we detect the op- tical signals time-integrated with an optical multichannel analyzer (OMA) after a spectrometer (with spectral resolution $0.1 \mathrm{meV}$ ), as a function of $\tau_{c c}$ and $\tau_{f w m}$, revealing the spectral information necessary for the interpretation of the $\mathrm{CC}$ in the inhomogeneous ensembles. Most of the experimental measurements have been performed on a wellcharacterized narrow $28 \AA$ GaAs single quantum well (SQW), surrounded by $250 \AA \mathrm{Al}_{0.3} \mathrm{Ga}_{0.7} \mathrm{As}$ barriers, grown by molecular-beam epitaxy with growth interruptions on the barrier-well interfaces. This leads to formation of large monolayer-plane islands with 11-monolayers (ML) thickness. ${ }^{13}$ The PL spectrum of this sample at $T=12 \mathrm{~K}$ consists of two peaks with an inhomogeneous linewidth $\sim 2.5 \mathrm{meV}$ and separation $\sim 13 \mathrm{meV}$, corresponding to the emissions from 10-ML and 11-ML regions, see Fig. 1(b). The resonant excitation on the high-energy side of the 10-ML resonance, see Fig. 1(b), allows for the simultaneous detection of the coherent and incoherent signals in the $\mathrm{CC}$ experiments. We note that the results reported here do not depend critically on the particular SQW and similar results are obtained from other multiple QW and superlattice samples with different ratios between the homogeneous and inhomogeneous broadenings.

\section{EXPERIMENTAL RESULTS}

Our main experimental results are shown in Figs. 1(c,d) with the spectrally resolved FWM signal for $\tau_{f w m}=0.5 \mathrm{ps}$, recorded around $\tau_{c c}=0 \mathrm{ps}$, and $\tau_{c c}=-3 \mathrm{ps}$ for 8 optical periods in the MI. In the direction of observation for the FWM signal, we simultaneously record PL from the sample on the low-energy side of the resonance (below $1.686 \mathrm{eV}$ outside of the laser excitation bandwidth). The strong fringes, corresponding to the optical period, of these optical signals are caused by the interference between the two polarizations excited by phase-locked CC laser pulses. For the FWM signal, the resulting polarization $P_{n e t}$, after the two $\mathrm{CC}$ pulses, is probed by the delayed laser pulse resulting in the observed time-integrated signal proportional to $P_{n e t}$. In this way, the FWM configuration is used to record a collimated signal proportional to $P_{n e t}$ but is otherwise not important for the conclusions of this paper. In fact, our results are comparable to other results obtained by recording resonant Rayleigh scattering proportional to $P_{n e t}$. For the photoluminescence signal, we observe fringes caused by the population modulation proportional to $\left|P_{n e t}\right|^{2}$ following energy relaxation to the emitting states on the low-energy side. The striking time-delay dependence of the coherent control signals is evident from Figs. 1(c,d). For $\tau_{c c}=0$ ps in Fig. 1(c) we find no spectral variation of the PL and FWM signals, whereas for $\tau_{c c}=-3$ ps in Fig. 1(d) the fringes in the FWM acquires a phase, dependent on the detected photon energy $\hbar \omega$, and the PL signal appears to be proportional to the spectrally integrated $P_{n e t}=P_{n e t}\left(\omega, \tau_{c c}\right)$ i.e., without any spectral variation. Indeed, in Fig. 2(a) we show how this phase change appears for the FWM signal and directly compare the spectrally integrated FWM signal with the PL signal demonstrating the proportionality between the two. On a longer time-delay scale in the $\mathrm{CC}$ experiment, we find much faster decay times for fringe contrast (FC) of the PL and spectrally 


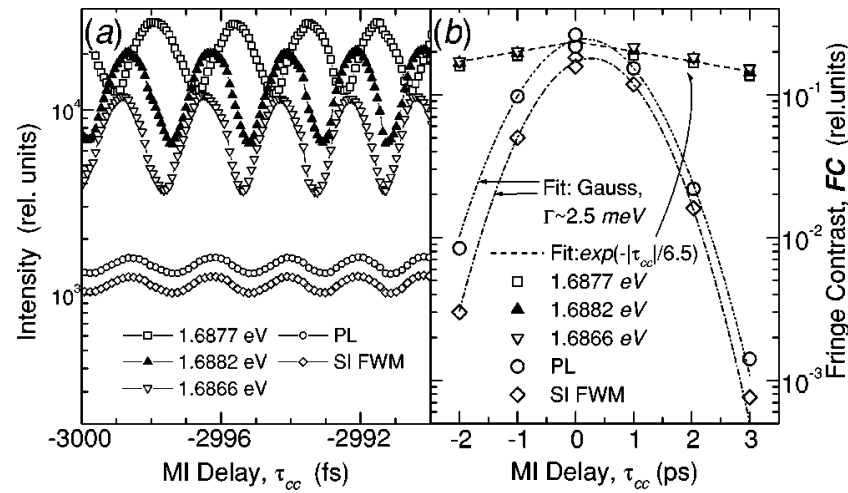

FIG. 2. (a) The FWM signal at photon energies 1.6877 (squares), 1.6882 (filled triangles), and 1.6866 (open triangles) eV, the spectrally integrated (1.686-1.690 eV) FWM signal (diamond), and the PL signal (circles) versus $\tau_{c c}$. (b) Corresponding decay of the fringe contrast with $\tau_{c c}$. The fits are exponential (dashed with decay time $6.5 \mathrm{ps}$ ) and Gaussian (dashed-dotted corresponding to $\Gamma=2.5 \mathrm{meV})$.

integrated FWM signals compared to the FC of the spectrally resolved FWM signals, see Fig. 2(b). Only the decay times for the fringe contrast in spectrally resolved FWM signals corresponds to the pure FWM-determined polarization decay times $T_{2}$ of the $10 \mathrm{ML}$ resonance, which is shorter than the previously determined polarization decay times of the $11 \mathrm{ML}$ resonance in the same sample. ${ }^{14}$ We can therefore conclude for inhomogeneous ensembles that the FC for spectrally resolved CC FWM signals decays with $T_{2}$ whereas the FC for spectrally-integrated CC signals such as PL or FWM decay as a result of the interplay between inhomogeneous and homogeneous broadening. In the following, we develop simple expressions for the FC describing how the inhomogeneous and homogeneous broadening, the time-delay $\tau_{c c}$, and the detection configuration (PL and FWM) determines the FC in $\mathrm{CC}$ experiments.

\section{THEORY}

The theoretical basis for spectrally resolved FWM has been presented in previous papers. ${ }^{15,16}$ In this paper, we discuss the extension of the theory to two levels (ground and excited states) with optical transitions, characterized by the frequency $\omega_{12}$ and the damping $\gamma_{12}=1 / T_{2}$, and excited by two phase-locked $\delta$ pulses: $E(t) \sim \delta(t) e^{-i \omega_{L} t}+a \delta(t$ $\left.-\tau_{c c}\right) e^{-i \omega_{L}\left(t-\tau_{c c}\right)}$ with the laser frequency $\omega_{L}$ and $t=0$ at the arrival time of the second pulse, i.e., the first pulse arrives at a delay $t=\tau_{c c}<0$, normally. The $a$ accounts for differences in the field strength, and the delta functions $\delta(t)$ are used for convenience in the short-pulse limit compared to $T_{2}$.

\section{A. Homogeneous broadening}

The first-order polarization $P_{\mathrm{hom}}^{(1)}\left(t, \tau_{c c}\right)$ induced by the phase-locked pulses for homogeneously broaded two-level systems is

$$
\begin{aligned}
P_{\text {hom }}^{(1)}\left(t, \tau_{c c}\right) \propto i N \mu_{21}^{2}\left[e^{-i \Omega_{21} t} \Theta(t)\right. & \\
& \left.+a e^{-i \Omega_{21}\left(t-\tau_{c c}\right)} \Theta\left(t-\tau_{c c}\right)\right],
\end{aligned}
$$

where we have used the complex frequency $\Omega_{21}=\omega_{12}$ $-i \gamma_{12}$, the density of levels $N$, the optical matrix element $\mu_{21}$, and the Heaviside function $\Theta(t)$ with $t=0$ as the arrival time of the second CC pulse. From Eq. (1) we define the fringe contrast $\mathrm{FC}$ for coherent control experiments detecting the time-integrated (i.e., spectrally integrated (SI)) reradiated emission from the polarization, ${ }^{18}$ with $I_{\text {max,min }}$ $=\max , \min \left[\int\left|P_{k_{1}}^{(1)}\left(t, \tau_{c c}\right)\right|^{2} d t\right]$ as

$$
F_{S I}^{(1)}\left(\tau_{c c}\right)=\frac{I_{\max }-I_{\min }}{I_{\max }+I_{\min }}=\frac{2 a e^{-\gamma_{21} \mid \tau_{c c}}}{1+a^{2}} .
$$

This shows that $F_{S I}^{(1)}\left(\tau_{c c}\right)$ decays with $T_{2}$ for increasing time separation between the CC pulses. A quantitatively similar expression is found when calculating the FC from the resonantly excited carrier density using a Bloch vector calculation. ${ }^{6}$ When the observed PL is proportional to the excited carrier density we can conclude that detecting the incoherent PL or the coherently reradiated emission in CC experiments reveals the same result.

Following Erland et al., ${ }^{16}$ the third-order polarization $P_{\text {hom }}^{(3)}\left(t, \tau_{f w m}, \tau_{c c}\right)$ emitting the FWM signal in the direction $2 k_{2}-k_{1}$, using Eq. (1) as the starting point, is

$$
\begin{aligned}
P_{\text {hom }}^{(3)}\left(t, \tau_{f w m}, \tau_{c c}\right) \propto & N \mu_{21}^{4}\left[\Theta\left(\tau_{f w m}\right)+a e^{-i \Omega_{21}^{*} \tau_{c c}}\right. \\
& \left.\Theta\left(\tau_{f w m}-\tau_{c c}\right)\right] e^{-i \Omega_{21}\left(t-\tau_{f w m}\right)} \\
& \times e^{i \Omega_{21}^{*} \tau_{f w m} \Theta\left(t-\tau_{f w m}\right),}
\end{aligned}
$$

where the "**" denotes complex conjugation and the Heaviside functions $\Theta(t)$ account for the emission of the FWM signal after the probe pulse. The dependence of the CC term in the square bracket in Eq. (3) on $\tau_{c c}$ is similar to the firstorder polarization in Eq. (1), proving the statement that the FWM signal as function of the phase-delay $\tau_{c c}$ is proportional to the first-order polarization. The spectrally resolved FWM is in this case:

$$
\begin{aligned}
P_{\text {hom }}^{(3)}\left(\omega, \tau_{f w m}, \tau_{c c}\right) \propto & N \mu_{21}^{4}\left[\Theta\left(\tau_{f w m}\right)\right. \\
& +a e^{\left.-i \Omega_{21}^{*} \tau_{c c} \Theta\left(\tau_{f w m}-\tau_{c c}\right)\right]} \frac{e^{i \Omega_{21}^{*} \tau_{f w m}}}{\Omega_{21}-\omega},
\end{aligned}
$$

showing that all the time-integrated spectral components have the same dependence on the phase-delay $\tau_{c c}$. We can therefore define the fringe contrast FC in FWM coherent control experiments from Eq. (4) in the following way: 


$$
\begin{aligned}
F_{S R, \text { hom }}^{(3)}\left(\tau_{c c}\right)=F_{S I, \text { hom }}^{(3)}\left(\tau_{c c}\right) & =\frac{\left|P^{(3)}\right|_{\max }^{2}-\left|P^{(3)}\right|_{\text {min }}^{2}}{\left|P^{(3)}\right|_{\max }^{2}+\left|P^{(3)}\right|_{\text {min }}^{2}} \\
& =\frac{2 a e^{-\gamma_{21}\left|\tau_{c c}\right|}}{e^{-2 \gamma_{21}\left|\tau_{c c}\right|}+a^{2}},
\end{aligned}
$$

with the decay of the $F_{\text {hom }}^{(3)}\left(\tau_{c c}\right)$ for increasing delay $\tau_{c c}$ according to the definition given above.

From Eqs. (2) and (5) it can be concluded that the coherent control signals for homogeneously broadened systems will decay with $T_{2}$ as a function of $\left|\tau_{c c}\right|$ under typical conditions with $a \sim 1$ and $\left|\tau_{c c}\right|>T_{2}$. Notice that a delayed maximum of the $F_{\text {hom }}^{(3)}$ at $\left|\tau_{c c \text {, } \max }\right|=\ln (a) / \gamma_{21}$ can be found when $a \neq 1$. The intuitive explanation of this is that the $F_{\text {hom }}^{(3)}$ is maximum when the remaining polarization amplitude due to the first CC pulse is equal to the polarization amplitude induced by the second CC pulse. This occurs for nonzero $\tau_{c c}$ when the intensity of the first pulse is largest, i.e., $a^{2}<1$. Notice also that $\max \left[F_{\text {hom }}\left(\tau_{c c, \max }\right)\right]=1$. The decay of the FC with $T_{2}$ is the interpretation of CC experiments first developed in atomic and molecular spectroscopy. In the following we will shown that this interpretation is not valid when inhomogeneous broadening plays a role as is often the case in semiconductors.

\section{B. Inhomogeneous broadening}

With inhomogeneous broadening, described by a Gaussian distribution $G\left(\omega_{21}\right)$ with central frequency $\omega_{21}^{c}$ and with a broadening parameter $\Gamma=\Gamma_{F W H M} / \sqrt{8 \ln (2)}$ scaled to the full width at half maximum $\Gamma_{F W H M}$, the first order polarization $P_{i n h}^{(1)}\left(t, \tau_{c c}\right)$ is

$$
\begin{aligned}
P_{i n h}^{(1)}\left(t, \tau_{c c}\right)= & \int P_{\text {hom }}^{(1)}\left(\omega_{21}\right) G\left(\omega_{21}\right) d \omega_{21} \\
\propto & N \mu_{21}^{2}\left[e^{-i \Omega_{21}^{c} t} e^{-\Gamma^{2} t^{2} / 2} \Theta(t)\right. \\
& \left.+a e^{-i \Omega_{21}^{c}\left(t-\tau_{c c}\right)} e^{-\Gamma^{2}\left(t-\tau_{c c}\right)^{2} / 2} \Theta\left(t-\tau_{c c}\right)\right],
\end{aligned}
$$

i.e., the first-order polarization decays faster because of the destructive interferens within the inhomogeneously broadened resonance. With Eq. (6) we find the spectrally integrated fringe contrast $F_{S I, i n h}^{(1)}$ as

$$
\begin{aligned}
F_{S I, i n h}^{(1)}\left(\tau_{c c}\right) & =\frac{2 a e^{-\Gamma^{2} \tau_{c c}{ }^{2} / 4} \operatorname{erfc}\left(\frac{\gamma_{21}}{\Gamma}+\Gamma \tau_{c c} / 2\right)}{\left(1+a^{2}\right) \operatorname{erfc}\left(\frac{\gamma_{21}}{\Gamma}\right)} \\
& \approx \frac{2 a e^{-\Gamma^{2} \tau_{c c}{ }^{2} / 4}}{\left(1+a^{2}\right)},
\end{aligned}
$$

where the erfc denotes the complementary error function. This shows that the decay of the fringe contrast in inhomogeneously broadened systems, detecting the reradiated emission, is determined solely by the inhomogeneous broadening $\Gamma$ for $\Gamma \gg \gamma_{21}$. Note furthermore that when expanding the erfc for $\Gamma \rightarrow 0$, the expression for the homogeneously broadened case in Eq. (5) is recovered. Thus, in semiconductor systems, with inhomogeneous broadening, the decay of the fringe contrast does not decay with the $T_{2}$ time. $^{11,12}$ Note also that $F_{S I, i n h}^{(1)}\left(\tau_{c c}\right)$ has a delayed maximum, depending on the ratio $\gamma_{21} / \Gamma$, that may explain the small phase delay of the PL signal compared to the FWM signal in Fig. 1(c).

A similar behavior is found for FWM-detected CC starting from the expression for the third-order polarization with inhomogeneous broadening,

$$
\begin{aligned}
P_{i n h}^{(3)}\left(t, \tau_{f w m}, \tau_{c c}\right) \propto & N \mu_{21}^{4}\left[\Theta\left(\tau_{f w m}\right)+a e^{-\left(i \Omega_{21}^{*}+\Gamma^{2}\left(t-2 \tau_{f w m}\right)\right) \tau_{c c}}\right. \\
& \left.\times e^{-\Gamma^{2} \tau_{c c}{ }^{2} / 2} \Theta\left(\tau_{f w m}-\tau_{c c}\right)\right] e^{-i \Omega_{21}^{c}\left(t-\tau_{f w m}\right)} \\
& \times e^{i \Omega_{21}^{c *} \tau_{f w m}} e^{-\Gamma^{2}\left(t-2 \tau_{f w m}\right)^{2} / 2} \Theta\left(t-\tau_{f w m}\right),
\end{aligned}
$$

where the last term is the third-order polarization with inhomogeneous broadening due to one $k_{1}$ pulse at $t=0$ followed by the $k_{2}$ pulse resulting in a photon echo. The CC term in the square bracket is the extra term caused by the phaselocked $k_{1}$ pulse pair. We immediately note that the CC term is now time dependent in contrast to the homogeneously broadened case Eq. (3), i.e., the different spectral components will behave differently in the CC FWM experiment. This will be transparent in the following expressions for the fringe contrast. For the spectrally integrated case, calculated like in the homogeneously broadened case, we find:

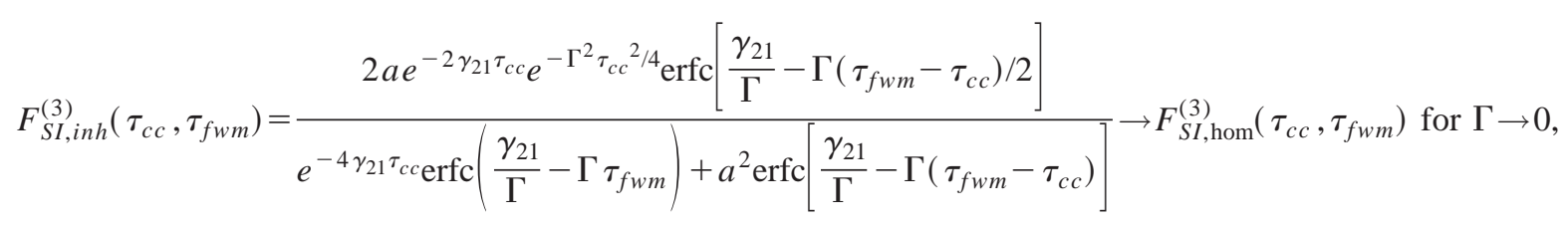

which is complicated by the three erfc expressions. However, as mentioned, the correct limit is found for $\Gamma \rightarrow 0$, whereas in the

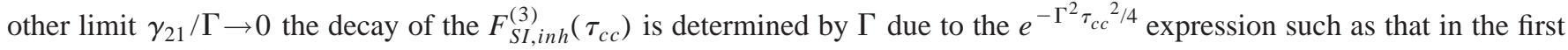
order case Eq. (7). This is used as the Gaussian fits in Fig. 2(b) determining the inhomogeneous broadening $\Gamma$. Equation (9) shows that the time-integrated CC FWM in general is a complicated interplay between the homogeneous broadening and the inhomogeneous broadening. Note that for finite $\tau_{c c} \neq 0$, the $F_{S I, \text { inh }}^{(3)}$ is always less than $1\left(F_{S I, \text { inh }}^{(3)}<1\right)$ for any $a$ and the 
so-called overshooting appearing at $F_{S I, i n h}^{(3)}=1$ is not possible in inhomogeneous systems. ${ }^{6}$ If the FWM signal is spectrally resolved, a different behavior, compared to the spectrally integrated case, is found in contrast to the homogeneously broadened case. First, the third-order polarization is found by a Fourier transformation of Eq. (8),

$$
\begin{aligned}
& P_{i n h}^{(3)}\left(\omega, \tau_{f w m}, \tau_{c c}\right) \propto N \mu_{21}^{4} \frac{\sqrt{\pi}}{\Gamma} e^{i 2 \omega \tau_{f}} e^{-2 \gamma_{21} \tau_{f}} e^{-\frac{\left(\Omega_{21}-\omega\right)^{2}}{2 \Gamma^{2}}}\left\{\Theta\left(\tau_{f w m}\right) \operatorname{erfc}\left[\frac{i\left(\Omega_{21}-\omega\right)}{\sqrt{2} \Gamma}-\frac{\Gamma \tau_{f w m}}{\sqrt{2}}\right]\right. \\
& \left.+a \Theta\left(\tau_{f w m}-\tau_{c c}\right) e^{-i \omega \tau_{c c}} e^{2 \gamma_{21} \tau_{c c} \operatorname{erfc}}\left[\frac{i\left(\Omega_{21}-\omega\right)}{\sqrt{2} \Gamma}-\frac{\Gamma\left(\tau_{f w m}-\tau_{c c}\right)}{\sqrt{2}}\right]\right\},
\end{aligned}
$$

where again only the $\mathrm{CC}$ term in the curly bracket determines the fringe contrast. We note that the period in the $\mathrm{CC}$ experiment is now determined by the detected frequency $\omega$ and different spectral components have different phases due the $\tau_{c c}$ term of the second erfc expression. From Eq. (10), we find the final general expression for the fringe contrast for spectrally resolved FWM $\mathrm{CC}$ experiments,

$$
\begin{aligned}
F_{S R, i n h}^{(3)}\left(\omega, \tau_{c c}, \tau_{f w m}\right) & =\frac{2 a e^{2 \gamma_{21} \tau_{c c}} \operatorname{erfc}\left[\frac{i\left(\Omega_{21}-\omega\right)}{\sqrt{2} \Gamma}-\frac{\Gamma\left(\tau_{f w m}-\tau_{c c}\right)}{\sqrt{2}}\right] \operatorname{erfc}\left[\frac{i\left(\Omega_{21}-\omega\right)}{\sqrt{2} \Gamma}-\frac{\Gamma \tau_{f w m}}{\sqrt{2}}\right]}{\left.\operatorname{erfc}\left[\frac{i\left(\Omega_{21}-\omega\right)}{\sqrt{2} \Gamma}-\frac{\Gamma \tau_{f w m}}{\sqrt{2}}\right]\right|^{2}+a^{2} e^{4 \gamma_{21} \tau_{c c}}\left|\operatorname{erfc}\left[\frac{i\left(\Omega_{21}-\omega\right)}{\sqrt{2} \Gamma}-\frac{\Gamma\left(\tau_{f w m}-\tau_{c c}\right)}{\sqrt{2}}\right]\right|^{2}} \\
& \approx \frac{2 a e^{-2 \gamma_{21} \tau_{c c}}}{e^{-4 \gamma_{21} \tau_{c c}+a^{2}}} \text { for fixed } \omega,
\end{aligned}
$$

i.e., each spectrally resolved component of the FWM signal will have a decay determined by $T_{2}$ in contrast to the spectrally integrated case Eq. (9). Based on Eq. (11), we evaluate a $T_{2}=13$ ps from the fits in Fig. 2(b) in agreement with pure FWM determinations (data not shown).

In Fig. 3 we present the calculated FWM spectra from Eq. (10) with $\hbar \Gamma=5,2.5,1.5$, and $0.75 \mathrm{meV}$ and $\hbar \gamma_{12}$ $=1 \mathrm{meV}\left(T_{2}=0.66 \mathrm{ps}\right)$ at a delay of $\tau_{f w m}=0.5 \mathrm{ps}$. The curves are calculated for different phase delays between the two pump pulses around $\tau_{c c}=-2.5$ ps resulting in firstorder polarizations with a phase differing by $0, \pi / 4,2 \pi / 4$, $3 \pi / 4$. Notice that the chosen $a=500$ compensates for the long $\tau_{c c}$ compared to $T_{2}$ to illustrate the interplay between the inhomogeneous and homogeneous broadenings. Notice also that this $a$ is smaller than the optimal $a_{\text {opt }}=e^{-2 \gamma_{21} \tau_{c c}}$ from Eq. (10). At small $\Gamma / \gamma_{21}$, the FWM spectra are Lorentzian except for a narrow central Gaussian part corresponding to $\Gamma$. Changing the relative phase between the two pump pulses, the Lorentzian changes the amplitude without noticable change in the shape of the FWM spectrum. At larger $\Gamma / \gamma_{21}$, the central Gaussian part becomes wider with more pronounced spectral modulations, while the remaining Lorentzian is without spectral modulations. From the plots of the largest $\Gamma / \gamma_{21}$ it becomes evident that the Gaussian part is spectrally modulated by $\Omega=2 \pi / \tau_{c c}$, while the modulation depth decreases again. This modulation depth is controlled by all the parameters in the FWM experiments i.e., $a, T_{2}, \Gamma$, $\tau_{f w m}$, and $\tau_{c c}$.

The significance of the spectral resolution is illustrated in Fig. 4 calculated from Eq. (10) for varying spectral resolution in the experiments. First we note that for $\tau_{f w m}=0$ ps the
FWM signal is identical to the coherent signal from the firstorder polarization $P^{(1)}$ showing that the FWM only act as a way to record the coherent emission from $P^{(1)}$ in the particular direction $2 k_{2}-k_{1}$. Otherwise the FWM technique for $\tau_{f w m}=0 \mathrm{ps}$ is similar to experiments recording, e.g., resonant Rayleigh scattering that is proportional to the induced

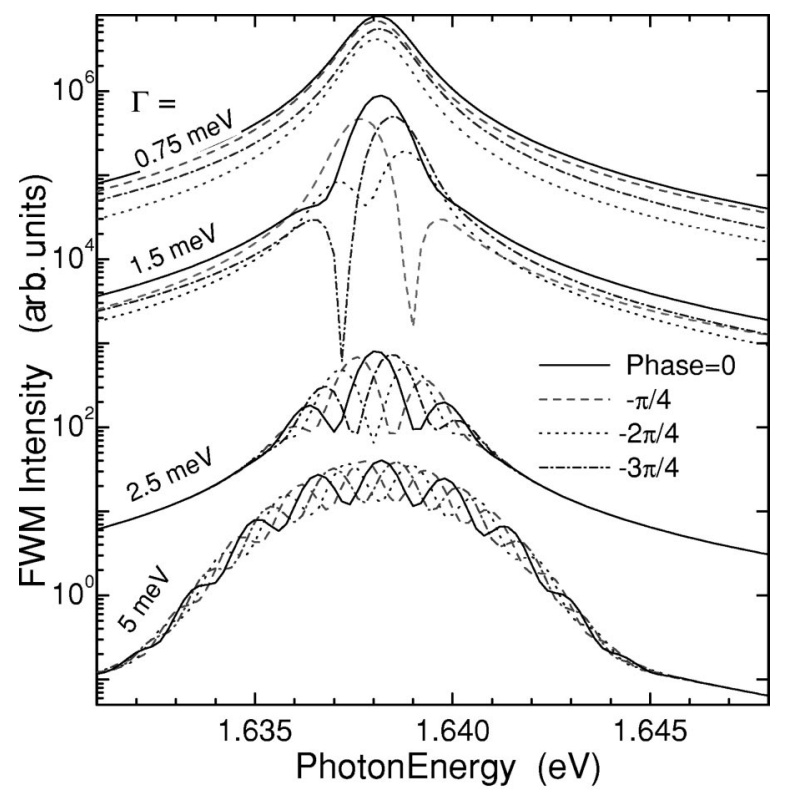

FIG. 3. Calculated FWM spectra for different inhomogeneous broadenings $\hbar \Gamma=0.75,1.5,2.5$, and $5 \mathrm{meV}$ using Eq. (2) with $\tau_{f w m}=0.5 \mathrm{ps}, \hbar \gamma_{21}=1 \mathrm{meV}, \tau_{c c}=-2.5 \mathrm{ps}$, and $a=500$. Solid, dashed, dotted, and dash-dotted curves are calculated for different relative phases between the phase-locked pump pulses. 


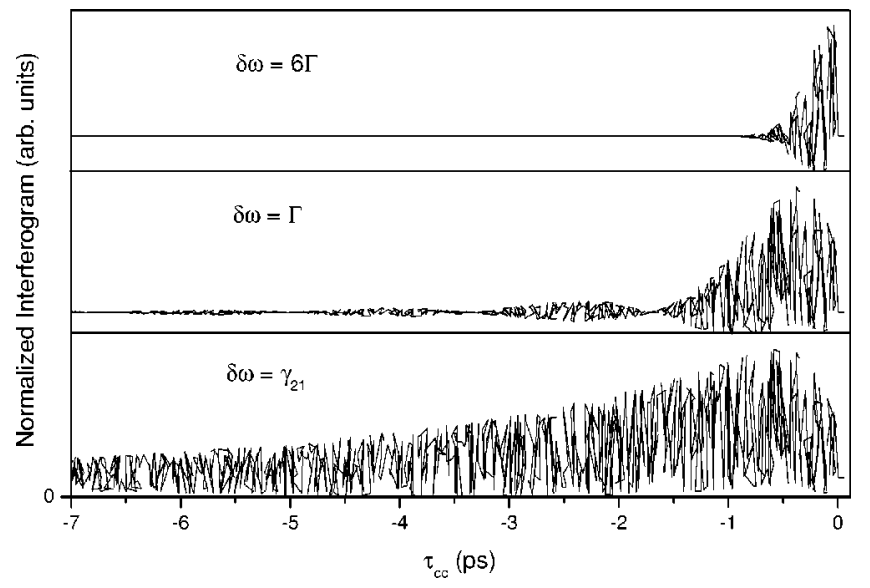

FIG. 4. Calculated interferograms from Eq. (2) showing the FWM signal versus $\tau_{c c}$ with $\hbar \Gamma=2.5 \mathrm{meV}, T_{2}=9 \mathrm{ps}$, and $a=1$ for different spectral resolution $\delta \omega$ in the $\mathrm{CC}$ experiment.

polarization $P^{(1)} \cdot{ }^{10}$ With the $\mathrm{CC}$, we find that the fringe contrast as a function of $\tau_{c c}$ decays very differently for different spectral filterings $\delta \omega$ because of the convolution with the spectral filter function $F_{S}(\omega, \delta \omega)=1, \omega \in \omega_{21}^{c} \pm \delta \omega / 2$ and $F_{S}(\omega, \delta \omega)=0, \omega \notin \omega_{21}^{c} \pm \delta \omega / 2 .{ }^{17}$ In Fig. 4 , we plot normalized interferograms, calculated as the integral over the spectral window $\delta \omega$ around $\omega_{21}^{c}, \quad I_{i n h}^{F W M}\left(\tau_{c c}, \delta \omega\right)$ $\propto\left|\int d \omega P_{i n h}^{(3)}\left(\omega, 0, \tau_{c c}\right) F_{S}(\omega, \delta \omega)\right|^{2}$, showing how the fringe contrast decays. For spectrally integrated detection, i.e. $\delta \omega$ $\gg \Gamma$, the fringe contrast decays on a time scale given by the inverse inhomogeneous broadening. In the other limit of spectrally resolved detection, i.e., $\delta \omega \leqslant \gamma_{21}$, the fringe contrast decays with $T_{2}$. In the intermediate case, the CC response as a function of $\tau_{c c}$ can be rather complex due to the finite spectral filter function $F_{S}(\omega, \delta \omega)$ as observed by Gurioli et al. ${ }^{10}$ In the following, we focus on the spectrally resolved CC with FWM to avoid complications from spectral filtering effects.

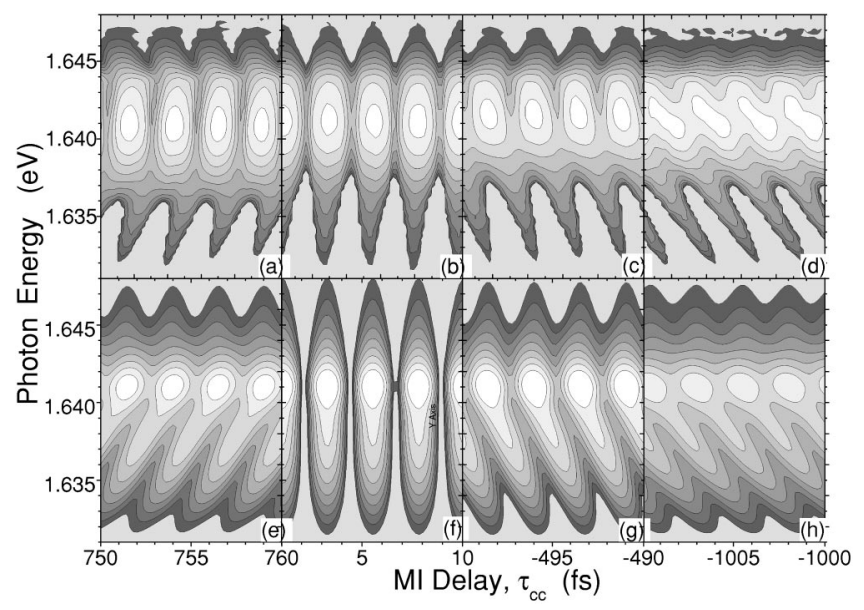

FIG. 6. Experimentally measured at $120 \mathrm{~K}(\mathrm{a}-\mathrm{d})$ and calculated $(\mathrm{e}-\mathrm{h})$ FWM spectra as a function of $\tau_{c c}$ around $0.75 \mathrm{ps}(\mathrm{a}, \mathrm{e}), 0 \mathrm{ps}$ $(\mathrm{b}, \mathrm{f}),-0.5 \mathrm{ps}(\mathrm{c}, \mathrm{g})$, and $-1 \mathrm{ps}(\mathrm{d}, \mathrm{h})$. The parameters for the calculation are given in the text.

\section{SPECTRALLY RESOLVED COHERENT CONTROL}

To obtain a better understanding of how the interplay between the inhomogeneous and homogeneous broadening enters into the spectrally resolved FWM as described by Eq. (10), we show in Fig. 5 how the FWM spectra transform with decreasing inhomogeneous broadening. The FWM spectra have been calculated with energies $\left(\hbar \omega_{21}^{c}\right.$ $=1.638 \mathrm{eV})$ and broadenings $\left(\hbar \gamma_{21}=0.75 \mathrm{meV}\right)$ relevant for the experimental results to be presented in Fig. 6. For large inhomogeneous broadening, we show in Fig. 5(a) that the different spectral components have different phase $\omega \tau_{c c}$ within the chosen spectral window, where the dynamic range of the FWM signal is more than three orders of magnitude. In the other limit of small inhomogeneous broadening, as already discussed, the FWM CC fringes have no spectral variation as shown in Fig. 5(f) for $\Gamma / \gamma_{21}=1.3$. However, in

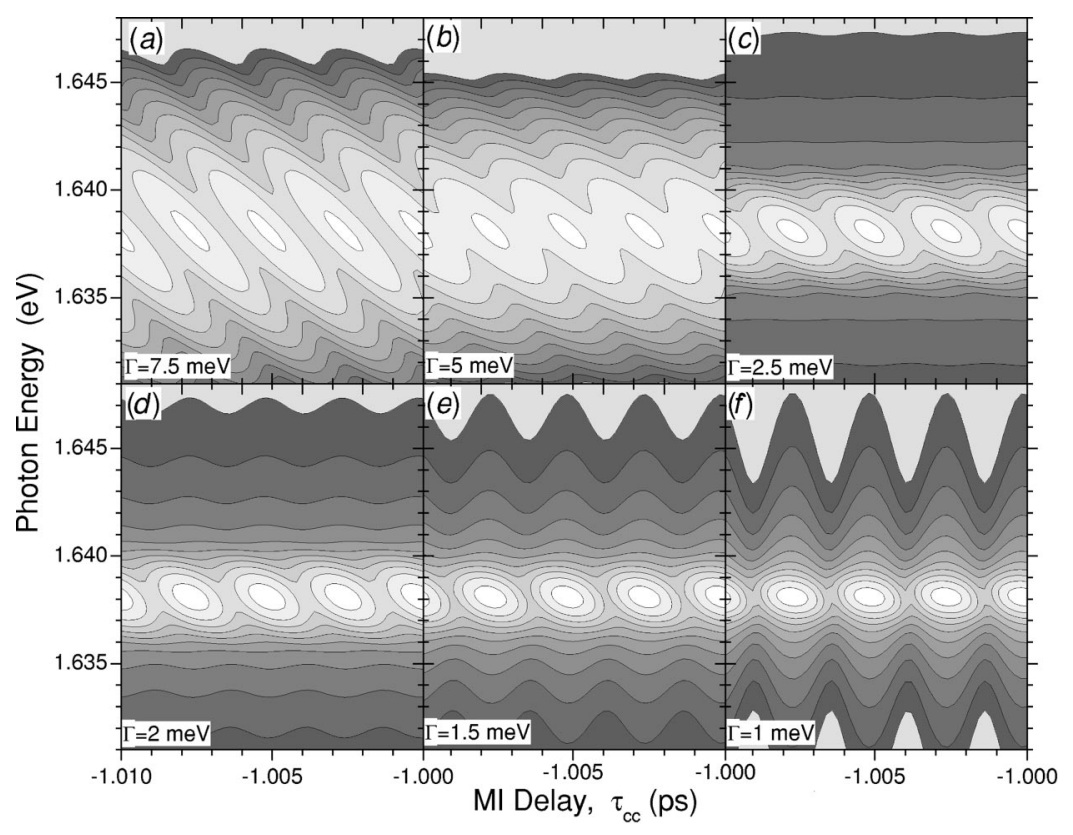

FIG. 5. Calculated FWM spectra with decreasing inhomogeneous broadening $(\mathrm{a}-\mathrm{f})$ with energy $\hbar \omega_{21}^{c}=1.638 \mathrm{eV}$ and $\gamma_{21}=0.75 \mathrm{meV}$ ver$\operatorname{sus} \tau_{c c}$. 
between these two limits we find that the spectral wings of the FWM spectra have the same phase, whereas an increasing part of the center acquires the phase change. This occurs as a result of the mixing of the Lorentzian part due to the homogeneous broadening, with constant phase $\omega_{21}^{c} \tau_{c c}$, and the Gaussian part due to the inhomogeneous broadening in the complex erfc expression in Eq. (10). In Fig. 5, around a phase delay of $\tau_{c c}=-1 \mathrm{ps}$ the spectral range of the Gaussian part coincides with $\Gamma$. We therefore conclude that the transformation of the FWM spectra with increasing inhomogeneous broadening is due to an increasing contribution of the Gaussian part with phase $\omega \tau_{c c}$ and not due to a change of the actual phases with inhomogeneous broadening. The spectral variation of the fringe contrast is thus a measure of the homogeneous (no variation) and inhomogeneous contributions to the electronic resonance.

We have applied this FWM CC technique in greater detail to investigate the nature of the SQW GaAs electronic resonance, discussed above, at a temperature of $120 \mathrm{~K}$, where the homogeneous broadening is expected to have a significant contribution. We note that at this high temperature the decay times in FWM experiments are normally too fast to obtain much dynamic information on, e.g., scattering on interface roughnesses in the quantum wells. From previous investigations of similar samples, it has been concluded that the interface roughness is well described by the bimodal roughness model. ${ }^{19,20}$ At low temperature, this results in a biexponential decay of the FWM signal from a slightly wider GaAs SQW grown under similar conditions as the one studied here. The biexponential decay, however, is untraceable at temperatures above $50 \mathrm{~K} .{ }^{21}$ Here we show that the FWM CC technique is very useful even at higher temperatures. The experimental results are presented in Figs. 6(a-d) showing the variation of the FWM spectra for different phase delays $\tau_{c c}$. The spectral components on the high-energy side in this case has a constant phase, whereas on the low-energy side shows the spectral variation of $\mathrm{CC}$ fringes. From this, we can immediately conclude that the electronic resonance is composed of at least two parts with different inhomogeneous broadenings in agreement with previous studies. ${ }^{19,21}$ From the calculated FWM spectra [Figs. 6(e-h)], fitted to the experimental data, we obtain an overall spectral and phase agreement between the experiment and the modeling with two separate electronic resonances without mutual interference. From this we extracted the following parameters: The resonance at $\hbar \omega_{21}^{c}$ $=1.639 \mathrm{eV}$ has $\Gamma=7 \mathrm{meV}$ and $\gamma_{21}=0.75 \mathrm{meV}$, while the one at $\hbar \omega_{21}^{c}=1.641 \mathrm{eV}$ has $\Gamma=0 \mathrm{meV}$ and $\gamma_{21}=2.5 \mathrm{meV}$. This demonstrates the importance of this $\mathrm{CC}$ technique to study electronic dynamics in semiconductor nanostructures, also at conditions that can be inaccesible by other techniques such as pure FWM.

Focusing on the phase-controlled FWM spectroscopy, we present in Fig. 7 a comparison of the experimentally measured $(\mathrm{a}-\mathrm{d})$ and calculated $(\mathrm{e}-\mathrm{h})$ FWM spectra as a function of probe delay $\tau_{f w m}$ for different delays between two phasecorrelated pump pulses $\tau_{c c}=0 \mathrm{ps} \quad(\mathrm{a}, \mathrm{e}) ;-1 \mathrm{ps} \quad(\mathrm{b}, \mathrm{f})$; -3 ps $(\mathrm{c}, \mathrm{g})$, and $-4 \mathrm{ps}(\mathrm{d}, \mathrm{h})$. The experimental FWM spectra have been recorded at $\mathrm{T}=12 \mathrm{~K}$. The best agreement between the experimental and calculated spectra and their dy-

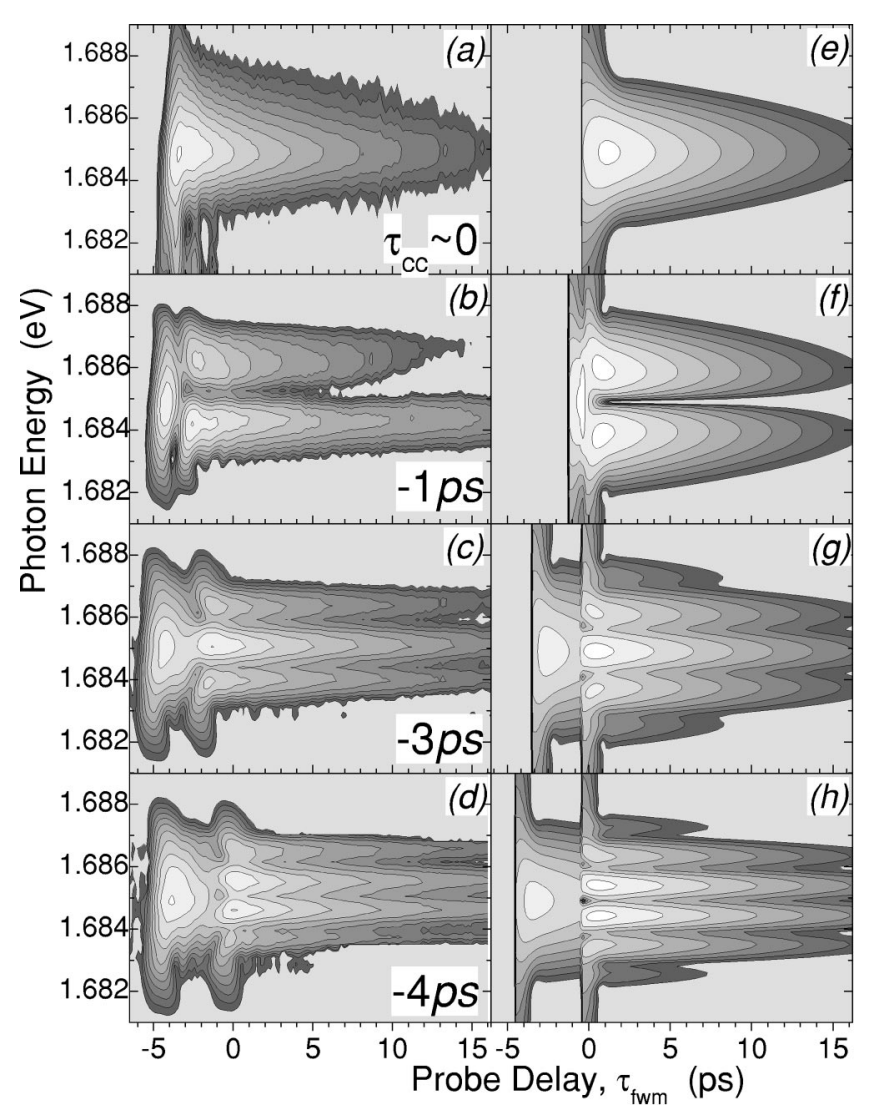

FIG. 7. Comparison of experimentally measured at $12 \mathrm{~K}(\mathrm{a}-\mathrm{d})$ and calculated $(\mathrm{e}-\mathrm{h}) \mathrm{FWM}$ spectra as functions of probe delay $\tau_{f w m}$ for different delays between the two phase-correlated pump pulses $\tau_{c c}=0 \mathrm{ps}(\mathrm{a}, \mathrm{e}),-1 \mathrm{ps}(\mathrm{b}, \mathrm{f}),-3 \mathrm{ps}(\mathrm{c}, \mathrm{g})$, and $-4 \mathrm{ps}(\mathrm{d}, \mathrm{h})$. In the calculation $\hbar \Gamma=2.5 \mathrm{meV}, \hbar \gamma_{21}=75 \mu \mathrm{eV}$, and $a=0.9$ were used.

namics was obtained with the following parameters: $\hbar \omega_{21}^{c}$ $=1.6849 \mathrm{eV}, \hbar \gamma_{21}=75 \mu \mathrm{eV}\left(T_{2}=9 \mathrm{ps}\right), \hbar \Gamma=2.5 \mathrm{meV}, a$ $=0.9$. One can see that without the second pump pulse or between the first and the second pump pulse, the FWM spectra are unmodulated and decay in accordance with the homogeneous and inhomogeneous broadenings of the excited excitonic states. After the second pump pulse (in our experimental conditions at $\tau_{f w m}>0$ ) the FWM spectra are strongly sinusoidally modulated with the Gaussian spectral shape split into several bands decaying with different rates. By fine tuning the MI delay $\tau_{c c}$, a maximum or a minimum of the modulated spectra can be shifted to any position in accordance with the interference term $\propto \cos \left(\omega \tau_{c c}\right)$ and $\Gamma / \gamma_{21} \gg 1$.

In Fig. 8, the experimentally measured at $\mathrm{T}=120 \mathrm{~K}$ (left panel) and calculated (right panel) FWM spectra for different delays between the phase-locked pump pulses $\tau_{c c}=0 \mathrm{ps}$ $(\mathrm{a}, \mathrm{e}),-0.75 \mathrm{ps}(\mathrm{b}, \mathrm{f}),-2,5 \mathrm{ps}(\mathrm{c}, \mathrm{g})$, and $-5 \mathrm{ps}(\mathrm{d}, \mathrm{h})$ are presented. The experimentally observed spectra are more asymmetric than the corresponding calculated spectra because of the non-Gaussian line shape due to the presence of localized exciton states on the low-energy side and a possible minor contribution from photoluminescence in the experiment. However, the calculated FWM spectra demonstrate similar pronounced modulations with a period and amplitude 


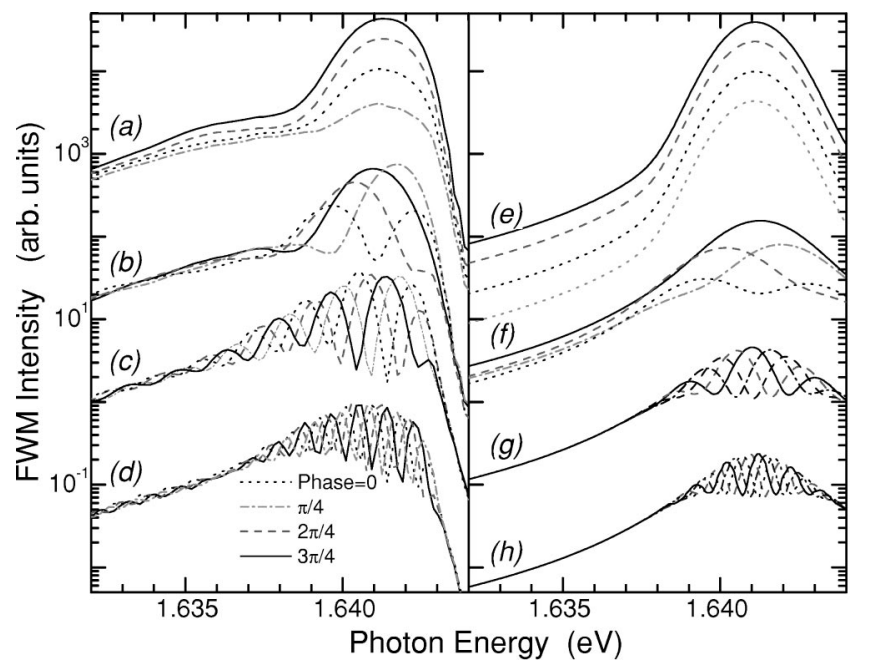

FIG. 8. Experimentally measured at $120 \mathrm{~K}$ (left panel) and calculated (right panel) FWM spectra at $\tau_{f w m}=1 \mathrm{ps}$ for different relative phases and different delays between the phase-locked pump pulses: $\tau_{c c}=0 \mathrm{ps}(\mathrm{a}, \mathrm{e}),-0.75 \mathrm{ps}(\mathrm{b}, \mathrm{f}),-2.5 \mathrm{ps}(\mathrm{c}, \mathrm{g})$, and $-5 \mathrm{ps}$ (d,h). In the calculation $\hbar \Gamma=2.75 \mathrm{meV}, \hbar \gamma_{21}=1.5 \mathrm{meV}$, and $a$ $=0.9$ were used.

strongly decaying with increasing $\tau_{c c}$. A comparison of the central parts of the experimental spectra with corresponding calculations allows us to determine the set of homogeneous and inhomogeneous broadenings, $\hbar \gamma_{21}=1.5 \mathrm{meV}\left(T_{2}\right.$ $=440 \mathrm{fs}$ ) and $\Gamma=2.75 \mathrm{meV}$, giving the impressive coincidence in spite of the difficult experimental conditions at $\mathrm{T}=120 \mathrm{~K}$ and $a=0.9$, where the dephasing time becomes very short. This spectroscopic technique is therefore very useful at higher temperatures, e.g., room temperature, where devices for optical communication operate with very short relaxation times. $^{22}$

\section{CONCLUSION}

In conclusion, we have taken inhomogeneous broadening into account for the applications of CC techniques to semiconductors. As we have demonstrated, the additional phase information with $\mathrm{CC}$ allows us to extract detailed information on broadenings in semiconductor samples. We have developed a model for the fringe contrast taking the optical phase into account with linear or nonlinear detection of the CC. In spectrally integrated experiments, the fringe contrast decays as an interplay between the inhomogeneous and homogeneous broadenings, whereas with spectral resolution, the full information on both the inhomogeneous and homogeneous broadenings can be obtained. Our main result is that inhomogeneous broadening leads to different phase-delay dependence for different spectral components, giving a direct measure of the inhomogeneous broadening. Based on that, we could confirm the bimodal roughness model for narrow quantum wells with growth interruptions. Thus, we have developed a basis for applications of CC techniques to semiconductor samples.

\section{ACKNOWLEDGMENTS}

We thank D. Gammon and D.S. Katzer, Naval Research Laboratory, Washington D.C., for providing one of the samples for this study and D. Birkedal for enlightning discussions. This work was supported by the Danish Natural Science Research Council. V.L. acknowledges support from Otto Mønsteds Foundation, RFBR and FNT.
${ }^{1}$ J.A. Yeazell and C.R. Stroud, Jr., Phys. Rev. Lett. 60, 1494 (1988).

${ }^{2}$ W.S. Warren, H. Rabitz, and M. Dahleh, Science 259, 1581 (1993).

${ }^{3}$ R.N. Zare, Science 279, 1875 (1998).

${ }^{4}$ T.C. Weinact, J. Ahn, and P.H. Bucksbaum, Phys. Rev. Lett. 80, 5508 (1998).

${ }^{5}$ C. Leichtle, W.P. Schleich, I. Sh. Averbukh, and M. Shapiro, Phys. Rev. Lett. 80, 1418 (1998).

${ }^{6}$ A.P. Heberle, J.J. Baumberg, and K. Köhler, Phys. Rev. Lett. 75, 2598 (1995).

${ }^{7}$ X. Marie, P. Le Jeune, T. Amand, M. Brousseau, J. Barrau, M. Paillard, and R. Planel, Phys. Rev. Lett. 79, 3222 (1997).

${ }^{8}$ N.H. Bonadeo, J. Erland, D. Gammon, D. Park, D.S. Katzer, and D.G. Steel, Science 282, 1473 (1998).

${ }^{9}$ M.U. Wehner, M.H. Ulm, D.S. Chemla, and M. Wegener, Phys. Rev. Lett. 80, 1992 (1998).

${ }^{10}$ M. Gurioli, F. Bogani, S. Ceccheroini, and M. Colocci, Phys. Rev. Lett. 78, 3205 (1997).

${ }^{11}$ M. Woerner and J. Shah, Phys. Rev. Lett. 81, 4208 (1998).

${ }^{12}$ N. Garro, M.J. Snelling, S.P. Kennedy, R.T. Phillips, and K.H. Ploog, Phys. Rev. B 60, 4497 (1999); N. Garro, M.J. Snelling,
S.P. Kennedy, R.T. Phillips, and K.H. Ploog, J. Phys. C 11, 6061 (1999).

${ }^{13}$ D. Gammon, E.S. Snow, B.V. Shanabrook, D.S. Katzer, and D. Park, Science 273, 87 (1996).

${ }^{14}$ X. Fan, T. Takagahara, J.E. Cunningham, and H. Wang, Solid State Commun. 108, 857 (1998).

${ }^{15}$ J. Erland, and I. Balslev, Phys. Rev. A 48, R1765 (1993).

${ }^{16}$ J. Erland, K.-H. Pantke, V. Mizeikis, V.G. Lyssenko, and J.M. Hvam, Phys. Rev. B 50, 15047 (1994).

${ }^{17}$ H. Stolz, Time-resolved Light Scattering from Excitons, Springer Tracts in Modern Physics Vol. 130 (Spring-Verlag, New York, 1994).

${ }^{18}$ D.S. Yee, K.J. Yee, S.C. Hohng, D.S. Kim, T. Meier, and S.W. Koch, Phys. Rev. Lett. 84, 3474 (2000).

${ }^{19}$ D. Gammon, B.V. Shanabrook, and D.S. Katzer, Phys. Rev. Lett. 67, 1547 (1991).

${ }^{20}$ D. Birkedal, V.G. Lyssenko, K.-H. Pantke, J. Erland, and J.M. Hvam, Phys. Rev. B 51, R7977 (1995).

${ }^{21}$ J. Erland, J.C. Kim, N.H. Bonadeo, D.G. Steel, D. Gammon, and D.S. Katzer, Phys. Rev. B 60, R8497 (1999).

${ }^{22}$ P. Borri, W. Langbein, J.M. Hvam, F. Heinrichsdorff, M.H. Mao, and D. Bimberg, IEEE Photonics Technol. Lett. 12, 594 (2000). 